\title{
Improved positioning algorithm based on linear constraints on scatterers
}

\author{
Fei Zhou*, Xin-yue Fan and Ting-Ying Chen
}

\begin{abstract}
Non-line-of-sight (NLOS) error is a bottleneck problem influencing positioning accuracy. However, a large number of scatterers distribute randomly in the surrounding of the mobile station (MS) in the dense multipath environment, such as urban. In most cases, there is no obstacle between scatterer and MS. So, the geographic information of scatterers around MS can be used to restraint NLOS errors and improve the positioning accuracy. If scatterer can be regarded as the virtual base station (BS), the precondition of the positioning algorithm is easier to satisfy than the traditional positioning algorithm, such as circle positioning algorithm. The algorithm proposed in this article selects suitable scatterers with linear constraints by analyzing the Doppler frequency shift of received signals which reflected by scatterers. Thereby, the selected scatterers and only two real BSs form a complete positioning system. In addition, because MS is motionless in most scenarios, BS must be moving to acquire the Doppler frequency shift. The algorithm proposed in this article is adjusted for the scenarios. And the scatterers with linear constraint can also be utilize fully. Simulation results show the algorithm proposed in this article in two different scenarios, not only simplifies the traditional algorithm, but also achieves the higher positioning accuracy.
\end{abstract}

Keywords: Linear constraint, Non-line-of-sight, Doppler frequency shift, Virtual base station

\section{Introduction}

Non-line-of-sight (NLOS) error badly influences the accuracy of wireless positioning technology in the dense multipath environment, such as urban. In recent years, some algorithms using the information of scatterer have been developed to restraint NLOS errors. Their advantages of performance have attracted the attention of many researchers. These algorithms are mainly divided into two categories. The first category of the positioning algorithm utilizes different scatterer models to obtain some statistic information, e.g., probability density function of time-of-arrival (TOA) or angle-of-arrival (AOA). These statistic information are used to compute parameters which related to positioning. Scatterer models commonly assume that scatterers distribute in the surrounding of mobile station (MS) or base station (BS) [1,2], giving an Elliptical scattering model which suited for the Microcellular communications system. The model assumed that scatterers uniformly distributed with the ellipse which the focus of ellipse are BS and MS [3] gives a

\footnotetext{
* Correspondence: zhoufei@cqupt.edu.cn

Chongqing Key Lab of Mobile Communications Technology, Chongqing University Posts and Telecommunication CQUPT, Chongqing, China
}

disk model which suited for the macrocell communications system. In the model, scatterers are assumed to distribute with the disk around MS, and BS is out of the disk $[4,5]$, giving a type of geographical scattering model with non-uniform distribution of scatterers, for example, Gaussian distribution scattering model, Conical scattering model, Eccentro scattering model, and so on. According to these models, the statistic function, such as AOA, power azimuth spectrum, TOA, and power delay spectrum, can be acquired. Finally, some positioning algorithms based on scatterer model can apply TOA, AOA, or other parameters to compute the position of target [6-8].

The second category of positioning algorithm is independent of scatterer model, and utilizes the position of scatterer to compute the position of MS. The key of these algorithms is that NLOS error can be transformed into fixed factors. And positioning accuracy mainly depends on the accuracy of measurement parameters. Thereby, it is possible to achieve high positioning accuracy of scatterers and MS [9,10], giving some scatterer positioning algorithms based on nonlinear least square theory. These algorithms utilize synthetically spatial-time- 
frequency information of received signal to compute the position of scatterer and MS. Because the received multipath signals reflected by scatterers include lots of information about scatterers, the position of scatterer can be computed in theory [11], giving a network grid search algorithm based on single reflection geometry model, which utilizes the information of multiple BS to restraint NLOS error. In [12-14], several possible results of positioning and partial information of scatterer can be acquired from the priori information of BSs, and the selection of the final correct result can be fulfilled by scatterer information. If $\mathrm{BS}$ can measure the $\mathrm{AOA}$ and the rate of AOA variation of the measurement signal from scatterer, the position of scatterer can be computed. So, the constraint relation among BS, scatterer, and MS can be designed and can also be used to restraint positioning error. In most cases, the scatterer is assumed to be static. If the position of scatterer is known, scatterer can be regarded as virtual BS. Thereby, a circle fitting algorithm can be proposed based on virtual BS. In fact, the relation among scatterers, MS and BS can be obtained by analyzing AOA and angle-of-departure of signal. Thereby, system of linear equations can be designed from TOA. Positioning algorithms based on the linear equations have much advantage. For example, scatters and MS can be positioned simultaneously. And positioning accuracy only depends on measurement parameter. But the constraint condition of the category algorithm is hard to satisfy. In addition, if we can successfully identify NLOS propagation or LOS propagation from related parameters, positioning accuracy would be improved very much. If the number of LOS is enough, positioning algorithms is independent of NLOS error $[15,16]$. However, in a real environment, the number of LOS is too small or zero. So, the category of algorithms is hard to apply in real application. The algorithm proposed in this article is based on the second category of positioning algorithm. Referring to the previous study, multiple scatterers and MS in the same line are assumed to have the same absolute Doppler frequency shifts. Many methods that utilize linear constraint between scatterers and MS are introduced in [10]. Three cases are commonly considered in positioning algorithm: (i) one scatterer and MS are in the same line; (ii) two scatterers and MS are in the same line; (iii) three or more scatterers and MS are in the same line. In the first case, if only one scatterer is in the same line with MS, it is impossible to acquire enough scatterer by multipath signal pairing. So, the positioning algorithm proposed in this article is not effective. The third case is studied by so many researchers. However, the second case is more general than the third case. Thereby, the second case is more valuable in implementing the proposed positioning algorithm. Due to high performance of line constraint positioning and inappeasable precondition, this article proposed an improved positioning algorithm using linear constraint on two BSs [17-20].

The remainder of this article is organized as follows. Section 2 presents and analyzes traditional positioning algorithm, which uses linear constraint on scatterers. The key points multipath pairing and TOA reconstruction are analyzed. Section 3 introduces an improved positioning algorithm based on linear constraint on only two scatterers. Section 4 gives a special and valuable case for the moving BS situation. Then the proposed positioning algorithm in Section 3 is adjusted to suit for the new case. Section 5 provides the simulation and results. Finally, conclusion and the further work are given.

\section{Traditional positioning algorithm using linear constraints on scatterers}

Positioning algorithms using linear constraints on scatterers are mainly based on the single reflect model. When scatterer and MS are in the same line, their Doppler frequency shifts on multipath signals are the same. Thus, these multipath signals can be extracted by filtering all received signals. Assuming that the coordinate of MS is $\left(x_{0}, y_{0}\right)$, and the polar coordinates of the scatterer is $\left(\rho_{k}, \theta_{k}\right), k=1,2, \ldots, N$, then the following linear Equations (1) and (2) are derived when the scatter and the MS are in the same line [21,22]:

$$
\begin{aligned}
& y_{0}=a x_{0}+b \\
& \mathbf{s}=a \mathbf{c}+b \mathbf{e}
\end{aligned}
$$

where $a$ and $b$ are real numbers; $\mathbf{s}$, c, and $\mathbf{e}$ are $N \times 1$ vectors, $s_{k}=\rho_{k} \sin \left(\theta_{k}\right), c_{k}=\rho_{k} \cos \left(\theta_{k}\right), e_{k}=1, k=1,2, \ldots, N$. By using the following equation, we can derive TOA of $l_{k}$ of the multipath signal reflected by $k$ th scatter:

$$
l_{k}=\rho_{k}+\sqrt{\left(\rho_{k} \cos \left(\theta_{k}\right)-x_{0}\right)^{2}+\left(\rho_{k} \sin \left(\theta_{k}\right)-y_{0}\right)^{2}}
$$

Equation (4) is then derived from Equation (3)

$$
x_{0}^{2}+y_{0}^{2}=l_{k}^{2}-2 \rho_{k}\left[l_{k}-x_{0} \cos \left(\theta_{k}\right)-y_{0} \sin \left(\theta_{k}\right)\right]
$$

By combining Equations (1), (2), and (4), we can obtain

$$
a P_{k}+2 b l_{k}-g c_{k}-h s_{k}=q_{k}
$$


where

$$
\begin{aligned}
c_{k} & =\cos \left(\theta_{k}\right), s_{k}=\cos \left(\theta_{k}\right), P_{k}=l_{k}^{2} \cos \left(\theta_{k}\right), q_{k} \\
& =l_{k}^{2} \sin \left(\theta_{k}\right), g=2 b x_{0}+a\left(x_{0}^{2}+y_{0}^{2}\right), h \\
& =2 b y_{0}-\left(x_{0}^{2}+y_{0}^{2}\right)
\end{aligned}
$$

Thus, from Equation (5)

$$
a h+g=2 b x_{0}+2 a b y_{0}
$$

By substituting the TOA of $l_{k}$ and the AOA of $\theta_{k}$, which is measured by BS, into Equation (5), we can obtain Equation (7)

$$
A X=B
$$

where

$$
\begin{aligned}
X & =[a, b, g, h]^{T}, B=\left[q_{1}, q_{2}, \ldots, q_{k}\right]^{T}, \\
A & =\left[\begin{array}{cccc}
P_{1} & l_{1} & -c_{1} & -s_{1} \\
P_{2} & l_{2} & -c_{2} & -s_{2} \\
\vdots & \vdots & \vdots & \vdots \\
P_{N} & l_{N} & -c_{N} & -s_{N}
\end{array}\right]
\end{aligned}
$$

We can solve Equation (7) by using the least square (LS) algorithm.

$$
X=\left(A^{T} A\right)^{-1} A^{T} B
$$

By substituting $a, b, g$, and $h$ into Equations (1) and (6), we can obtain the position $\left(x_{0}, y_{0}\right)$ of MS.

$$
\left\{\begin{array}{l}
x_{0}=\frac{a h+g-2 a b^{2}}{2 b\left(1+a^{2}\right)} \\
y_{0}=\frac{a\left(a h+g-2 a b^{2}\right)}{2 b\left(1+a^{2}\right)}+b
\end{array}\right.
$$

\section{Improved positioning algorithm based on linear constraints on scatterers in two BSs}

Figure 1 shows a brief frame of the improved algorithm, which comprises three steps.

Step 1: Multipath signal pairing: The BS receives the multipath signal and measures the Doppler frequency shift of the signal. The signals that reflected from scatterers to BS are then paired. Two scatterers that lie on the same line with MS are selected.

Step 2: TOA reconstruction: After multipath signal pairing and scatterer selection, the chosen scatterers can be positioned based on the AOA

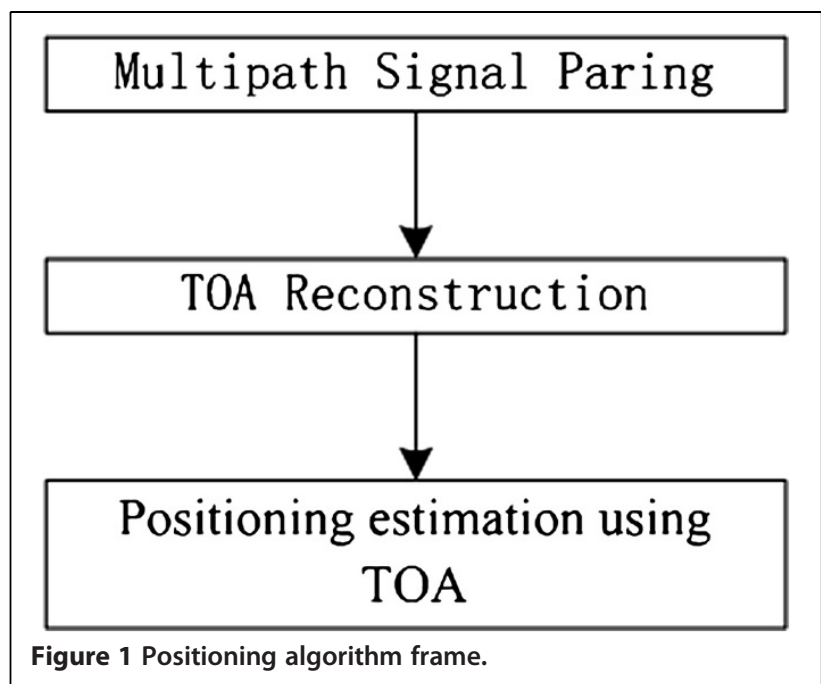

measured by the two BSs. The distances between MS and the two scatterers can be computed. Finally, by applying the linear constraints of two scatterers, we can implement TOA reconstruction.

Step 3: Positioning Estimation Using TOA: Two scatterers are regarded as virtual BS. At the same time, the positions of two real BSs are known, four TOAs are obtained. Some existing algorithms can be used to compute for the position of the MS.

\subsection{Multipath signal pairing}

If two scatterers and one MS are in the same line, then the multipath components, the Doppler frequency shifts, reflected from two scatterers and received by a certain BS are all equivalent. Thus, the key to multipath signal pairing is distinguishing multipath signals with the same Doppler frequency shifts received from the same BS. As shown in Figure 2, the position parameter $\left(x_{s i} y_{s i}\right)$ of the $i$ th scatterer is acquired by using Equation (10)

$$
\begin{aligned}
x_{S i} & =\frac{x_{2}-x_{1} \tan \beta_{2 i} \tan \beta_{1 i}+\left(y_{1}-y_{2}\right) \tan \beta_{2 i}}{1-\tan \beta_{1 i}} \\
y_{S i} & =\left(x_{S i}-x_{1}\right) \tan \beta_{1 i}+y_{1}
\end{aligned}
$$

where $\left(x_{1}, y_{1}\right)$ and $\left(x_{2}, y_{2}\right)$ are the position parameters of two BSs, and $\beta_{j i}$ is the direction of arrival of the $i$ th scatterer measured by the $j$ th BS. So, $\beta_{1 i}$ and $\beta_{2 i}$ can be obtained by Equation (11)

$$
\begin{aligned}
\tan \beta_{1 i} & =\frac{x_{S i}-x_{1}}{y_{S i}-y_{1}} \\
\tan \beta_{2 i} & =\frac{x_{S i}-x_{2}}{y_{S i}-y_{2}}
\end{aligned}
$$




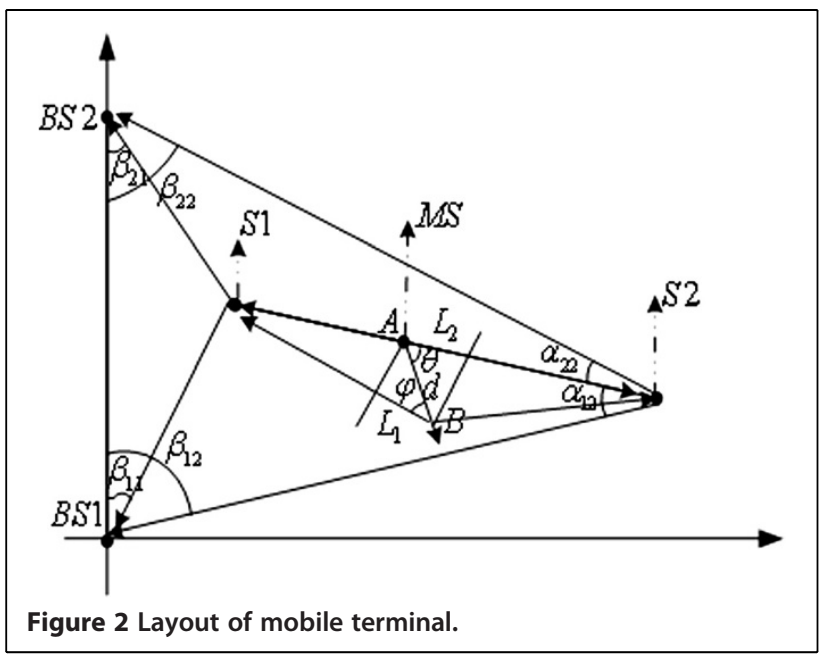

The linear distance between the $i$ th $\mathrm{BS}$ and the $j$ th BS is derived by Equation (12).

$$
L_{j i}=\sqrt{\left(x_{S i}-x_{j}\right)^{2}+\left(y_{S i}-y_{j}\right)^{2}}
$$

If the measurement error is zero, then Equation (13) is logical.

$$
L_{i}=l_{1 i}-L_{1 i}=l_{2 i}-L_{2 i}
$$

where $l_{j i}$ is TOA between $j$ th scatter and $i$ th BS. Equation (14) is the measurement error.

$$
E N=\left[\left(l_{2 i}-L_{2 i}\right)-\left(l_{1 i}-L_{1 i}\right)\right]^{2}
$$

Two datasets are paired when $E N$ is close to minimum. Thus, four datasets from two BSs $\left(l_{1 i}, \beta_{1 i}\right)$ and $\left(l_{2 i}, \beta_{2 i}\right),\left(l_{1 i}, \beta_{1 i}\right)$ and $\left(l_{2 j}, \beta_{2 j}\right),\left(l_{1 j}, \beta_{1 j}\right)$ and $\left(l_{2 i}, \beta_{2 i}\right)$, and $\left(l_{1 j}, \beta_{1 j}\right)$ and $\left(l_{2 j}, \beta_{2 j}\right)$, are grouped into four pairs. The ENs of the datasets can be computed using Equation (14). The dataset with the smallest EN must be reflected by the same scatterer and must be measured by two BSs separately; that is, the set must have correct pairing. So, the other sets of data measured by the same two BSs are also correct pairing.

\subsection{TOA reconstruction}

If the multipath signals of the different BSs are successfully paired, then the positioning parameter of scatterers $S_{i}$ and $S_{j},\left(x_{s i}, y_{s i}\right)$ and $\left(x_{s j}, y_{s j}\right)$ can be computed by using Equation (10). The distance between the two scatterers can be derived by using Equation (15).

$$
L_{S}=\sqrt{\left(x_{S i}-x_{S j}\right)^{2}+\left(y_{S i}-y_{S j}\right)^{2}}
$$

Thus, the line relation among $S_{i}, S_{j}$, and MS can be determined by $L_{i}, L_{j}$, and $L_{s}$.
If $L_{j}>L_{s}$ and $L_{j}>b L_{i}$, then $S_{i}$ is located between $S_{j}$ and MS.

If $L_{i}>L_{s}$ and $L_{i}>L_{j}$, then $S_{j}$ is located between $S_{i}$ and MS.

If $L_{s}>L_{i}$ and $L_{s}>L_{j}$, then MS is located between $S_{j}$ and $S_{i}$.

In case (1), $S_{i}, S_{j}$, and BS1 form a triangle. Thus, Equation (16) can be derived.

$$
\cos \alpha_{1 j}=\frac{L_{S}^{2}+L_{1 j}^{2}-L_{1 i}^{2}}{2 L_{S} L_{1 j}}
$$

In another triangle formed by $S_{j}$, MS, and BS1, the line distance $L_{\text {line1 }}$ between MS and BS1 can be obtained by using Equation (17).

$$
L_{\text {line } 1}=\sqrt{r_{j}^{2}+L_{1 j}^{2}-2 r_{j} L_{1 j} \cos \alpha_{1 j}}
$$

Similarly, the distance $L_{\text {line2 }}$ between MS and BS2 can be derived by Equation (18).

$$
L_{\text {line } 2}=\sqrt{r_{j}^{2}+L_{2 j}^{2}-\frac{\left(L_{S}^{2}+L_{2 j}^{2}-L_{2 i}^{2}\right) r_{j}}{L_{S}}}
$$

In case (2), on the basis of the triangle relations among $S_{i}, S_{j}$, and BSi $(i=1,2)$, we can calculate for $L_{\text {line1 }}$ and $L_{\text {line2 }}$ by using Equation (19). Thus, TOA reconstruction can be implemented.

$$
\begin{aligned}
& L_{\text {line } 1}=\sqrt{r_{i}^{2}+L_{1 i}^{2}-\frac{\left(L_{S}^{2}+L_{1 i}^{2}-L_{1 j}^{2}\right) r_{i}}{L_{S}}} \\
& L_{\text {line } 2}=\sqrt{r_{i}^{2}+L_{2 i}^{2}-\frac{\left(L_{S}^{2}+L_{2 i}^{2}-L_{2 j}^{2}\right) r_{i}}{L_{S}}}
\end{aligned}
$$

Similarly, the resolution of cases (1) and (2) is still in effect in case (3).

\subsection{Positioning parameter of mobile computation}

Figure 2 shows that $S_{i}$ and $S_{j}$ can be considered as virtual BS, and $r_{i}$ and $r_{j}$ are the TOAs in the line-of-sight (LOS) path of an MS to two scatterers $S_{i}, S_{j}$. The TOA in the LOS of BS1 and BS2 can be reconstructed. Thus, four TOAs from different BSs to MSs can be utilized to compute the positioning parameter of MS. The LS algorithm is the preferred algorithm.

$$
\left(x_{j}-x_{1}\right) x+\left(y_{j}-y_{1}\right) y=\frac{1}{2}\left[x_{j}^{2}+y_{j}^{2}-\left(x_{1}^{2}+y_{1}^{2}\right)+r_{1}^{2}-r_{j}^{2}\right]
$$


where $r_{j}^{2}=\left(x_{j}-x\right)^{2}+\left(y_{j}-y\right)^{2}$. Equation (20) can be derived and represented by the following vector matrix form, Equation (21)

$$
A X=B
$$

where

$$
\begin{aligned}
& X=\left[\begin{array}{l}
x \\
y
\end{array}\right] \\
& A=\left[\begin{array}{cc}
x_{2}-x_{1} & y_{2}-y_{1} \\
x_{S i}-x_{2} & y_{S i}-y_{2} \\
x_{S j}-x_{S i} & y_{S j}-y_{S i}
\end{array}\right] \\
& B=\left[\begin{array}{c}
\frac{1}{2}\left[x_{2}^{2}+y_{2}^{2}-\left(x_{1}^{2}+y_{1}^{2}\right)+L_{\text {line } 1}^{2}-L_{\text {line } 2}^{2}\right] \\
\frac{1}{2}\left[x_{S i}^{2}+y_{S i}^{2}-\left(x_{2}^{2}+y_{2}^{2}\right)+L_{\text {line } 2}^{2}-r_{i}^{2}\right] \\
\frac{1}{2}\left[x_{S j}^{2}+y_{S j}^{2}-\left(x_{S i}^{2}+y_{S i}^{2}\right)+r_{i}^{2}-r_{j}^{2}\right]
\end{array}\right]
\end{aligned}
$$

The position of MS can also be computed by using the LS algorithm, as shown in Equation (8).

\section{Improved algorithm on scatter linear restriction based on moving BS}

The above algorithm is inefficient when the MS is static, because the Doppler frequency shift cannot be obtained. However, the Doppler frequency shift can be obtained if the BS is mobile. Thereby, a novel positioning algorithm based on mobile BS is proposed by utilizing two multipath signals. The following are the basic steps of the proposed algorithm:

(1)The BS receives a multipath signal and measures the Doppler frequency shift of that signal. Signals reflected from scatterers to BS are then paired. Among the pairings, two scatterers that lie in the same line with the MS are chosen.

(2) After the paired multipath signal that is reflected by two scatterers is chosen, the positions of the paired signal can be computed by utilizing the circle fitting algorithm $[7,8]$ on the basis of the trajectory information of a single BS, TOA, and AOA.

(3) The TOA is reconstructed in each measurement point of any mobile trajectory of the BS.

(4) The measurement points of two scatterers and BS in the mobile trajectory are regarded as virtual BSs, and the reconstructed TOA is the TOA of LOS.

Thus, the position of the MS can be computed by employing the LS algorithm. The improved algorithm has two key points: multipath signals pairing and TOA/ AOA reconstruction.

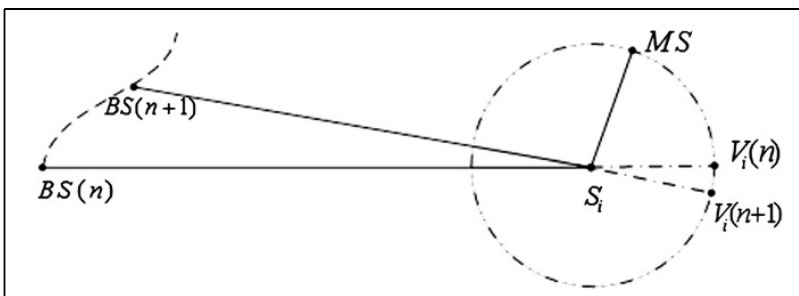

Figure 3 Circle motion trajectory of a pseudo-target.

\subsection{Scatterer position estimation by circle fitting algorithm \\ 4.1.1 Assumption}

During the entire motion estimation, the positions of the two scatterers are regarded as scatter points and are assumed stationary.

Considering that the measured distance is greater than the perturbation motion of the scatterer, this perturbation is regarded as the measurement error of TOA. Therefore, the above assumption is reasonable, and scatterers have transient stationary characteristics. Assuming that $N$ times measurement is executed in the motion trajectory of BS, as shown in Figure 3, the pseudo-target coordinate of the $n$th point $V(n)=\left(x_{i}(n), y_{i}(n)\right)$ can be computed by using Equation (25).

$$
\left\{\begin{array}{l}
x_{i}(n)=x_{n}+l_{i}(n) \cos \left(\alpha_{i}(n)\right) \\
y_{i}(n)=y_{n}+l_{i}(n) \sin \left(\alpha_{i}(n)\right)
\end{array}\right.
$$

Here, $\left(x_{n}, y_{n}\right)$ is the coordinate of the $n$th measurement point, $l_{i}(n)$ is the TOA of the measurement point, and $\alpha_{i}(n)$ is the AOA of the measurement point.

The MS shown in Figure 3 is a real mobile terminal. The MS is stationary during the entire process; thus, regardless of the motion trajectory of the BS, the distance $r_{i}$ between the pseudo-target $V_{i}(n)$ to the measurement point $\mathrm{BS}(n)$ and the $i$ th scatterer is the same. The $n$th pseudo-target to the measurement point $\mathrm{BS}(n)$ and MS are in the same circle, and the center of the circle is the

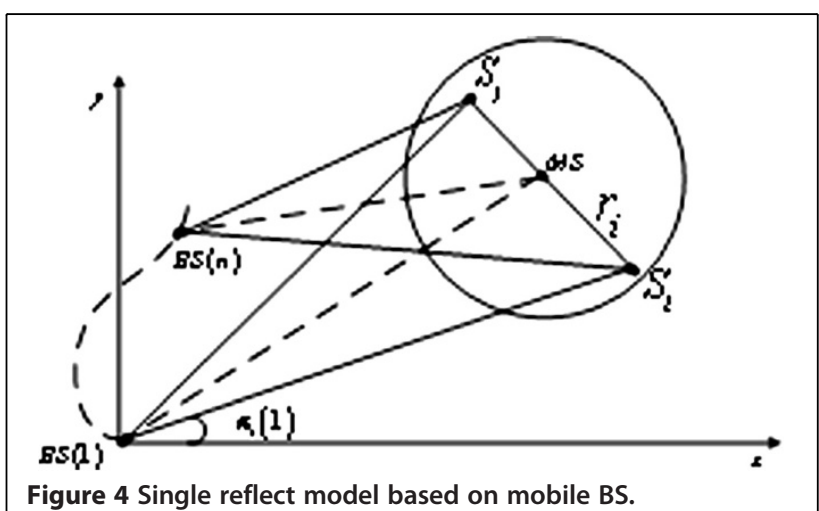


Table 1 Simulation parameters

\begin{tabular}{ll}
\hline Coordinate of $\mathbf{M S}$ & $\mathbf{( 8 0 0 , 8 0 0 )}$ \\
\hline Velocity along the $x$-axis of MS & $10 \mathrm{~m} / \mathrm{s}$ \\
\hline Velocity along the $y$-axis of MS & $10 \mathrm{~m} / \mathrm{s}$ \\
\hline Simulation number & 2000 \\
\hline Multipath number & 2 \\
\hline
\end{tabular}

$i$ th scatterer. The coordinate and radius of the circle are $\mathrm{S}_{i}=\left(x_{s i}, y_{s i}\right)$ and $r_{i}$, respectively.

$$
\left(x_{i}(n)-x_{s i}\right)^{2}+\left(y_{i}(n)-y_{s i}\right)^{2}=r_{i}^{2}
$$

Equation (27) can be derived by using the LS algorithm:

$$
A_{i} X_{i}=B_{i}
$$

where

$$
\begin{aligned}
A_{i}= & {\left[\begin{array}{ccc}
x_{i}(1) & y_{i}(1) & 1 \\
x_{i}(2) & y_{i}(2) & 1 \\
\vdots & \vdots & \vdots \\
x_{i}(N) & y_{i}(N) & 1
\end{array}\right], } \\
B= & {\left[\begin{array}{c}
x_{i}^{2}(1)+y_{i}^{2}(1) \\
x_{i}^{2}(2)+y_{i}^{2}(2) \\
\vdots \\
x_{i}^{2}(N)+y_{i}^{2}(N)
\end{array}\right], X_{i}=\left[\begin{array}{c}
-2 x_{s i} \\
-2 y_{s i} \\
x_{s i}^{2}+y_{s i}^{2}-r_{i}^{2}
\end{array}\right] }
\end{aligned}
$$

When the number of measurement points is not less than three, the solution of the LS algorithm is as follows:

$$
X_{i}=\left(A_{i}^{T} A_{i}\right)^{-1} A_{i}^{T} B_{i}
$$

Thus,

$$
\left\{\begin{aligned}
x_{s i} & =-X_{i}(1) / 2 \\
y_{s i} & =-X_{i}(2) / 2 \\
r_{i} & =\sqrt{X_{i}^{2}(1)+X_{i}^{2}(2)-4 X_{i}(3)} / 2
\end{aligned}\right.
$$

\subsection{TOA reconstruction of measurement point}

The two scatter positioning coordinates $\left(x_{s i}, y_{s i}\right)$ and $\left(x_{s j}\right.$, $\left.y_{s j}\right)$ as well as the distances $r_{i}$ and $r_{j}$ between two scatterers and MS can be calculated by using Equation (29). The coordinate of the $N$ measurement points can be computed by utilizing the initial coordinate and mobile trajectory during BS motion. The distance $L_{s}$ between two scatterers can be derived by the following equation:

$$
L_{S}=\sqrt{\left(x_{S i}-x_{S j}\right)^{2}+\left(y_{S i}-y_{S j}\right)^{2}}
$$

If $L_{i}>L_{s}$ and $L_{i}>L_{j}$, then the LOS distance of the $n$th measurement point $L_{n}^{\text {los }}$ can be obtained from Equation (31).

$$
\begin{gathered}
\left.L_{n}^{\text {Los }}=\left(\sqrt{r_{i}^{2}+\left(l_{i}(n)-r_{i}\right)^{2}-\left(\frac{\left(L_{S}^{2}+\left(l_{i}(n)-r_{i}\right)^{2}\right.}{\left.-\left(l_{j}(n)-r_{j}\right)^{2}\right) r_{i}}\right.}\right)\right) \\
a h+g=2 b x_{0}+2 a b y_{0}
\end{gathered}
$$

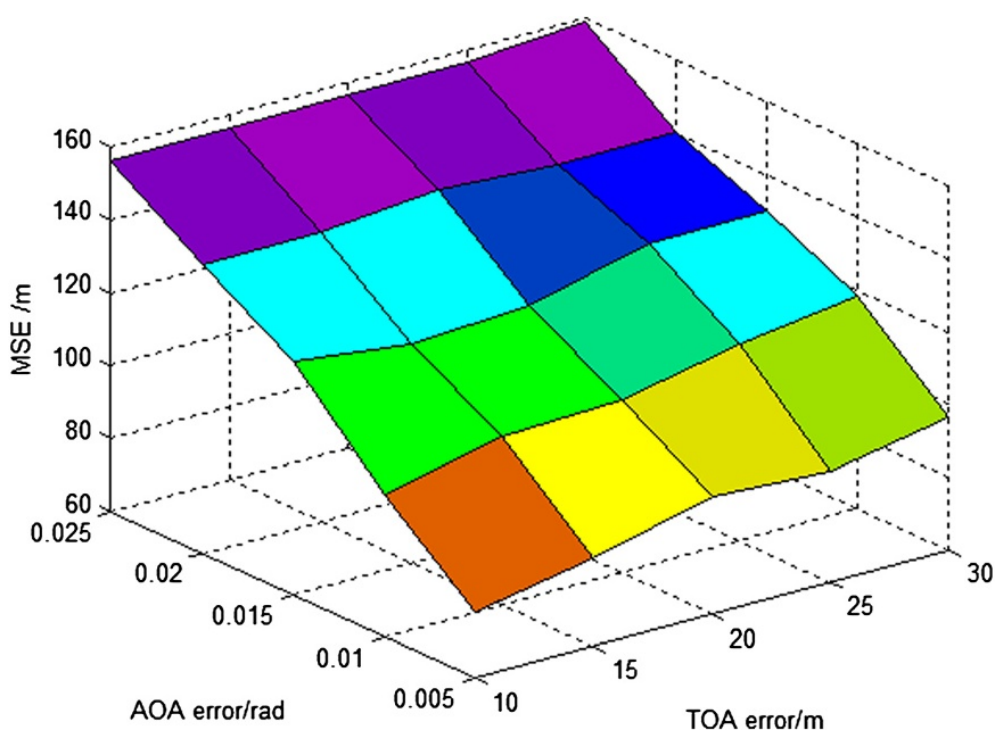

Figure 5 MSE of the different AOA and TOA measurement errors. 


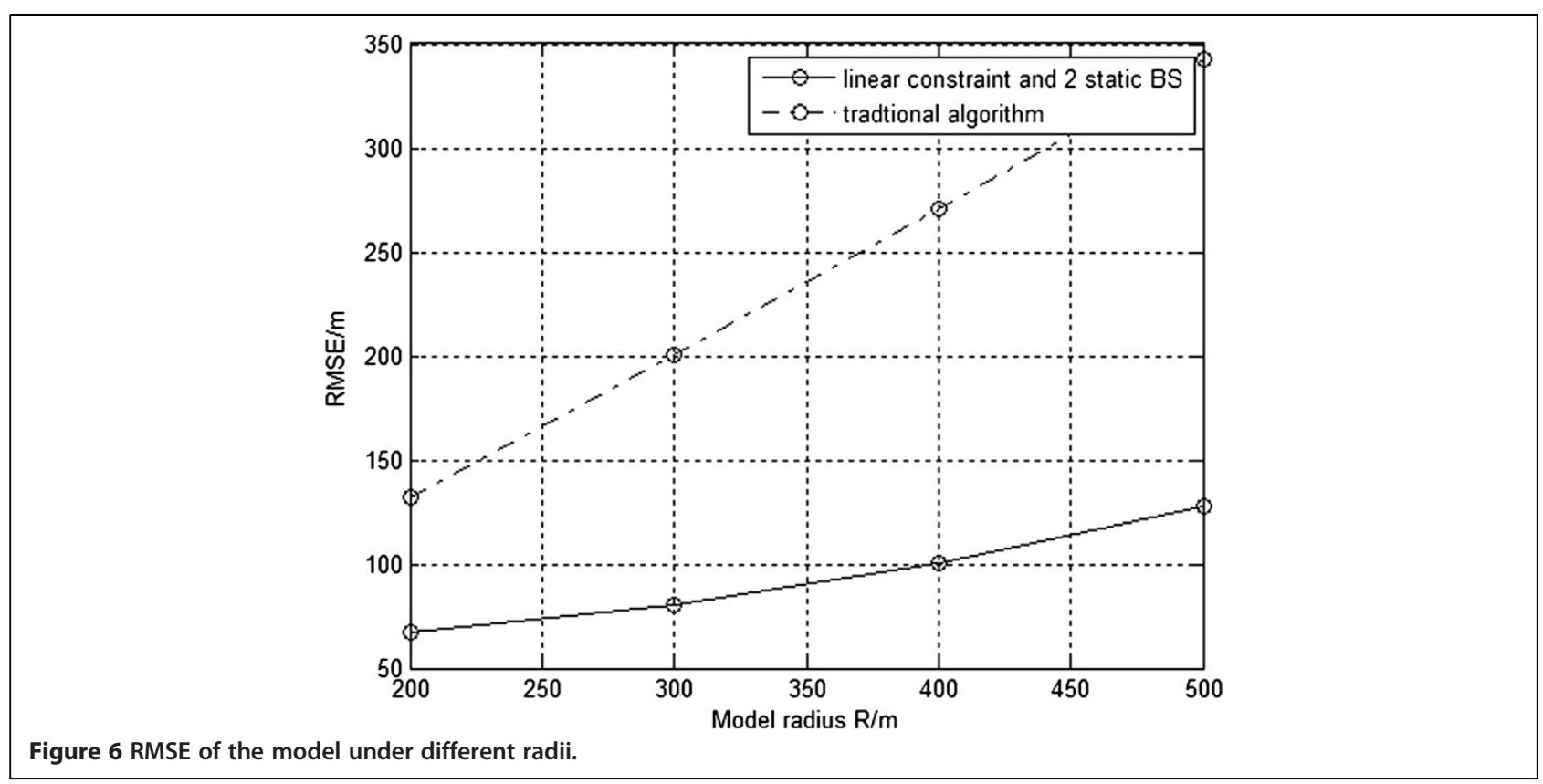

If $L_{j}>L_{s}$ and $L_{j}>L_{i}$, then the LOS distance of the $n$th measurement point $L_{n}^{L O S}$ can be obtained from Equation (32).

$$
L_{n}^{L o s}=\sqrt{r_{j}^{2}+\left(l_{j}(n)-r_{j}\right)^{2}-\left(\frac{\left(L_{S}^{2}-\left(l_{i}(n)-r_{i}\right)^{2}+\left(l_{j}(n)-r_{j}\right)^{2}\right) r_{j}}{L_{S}}\right)}
$$

If $L_{s}>L_{i}$ and $L_{s}>L_{j}$, then $L_{n}^{L O S}$ can be obtained by Equations (31) or (32).

\subsection{Computation of MS position}

In Figure $4, S_{i}$ and $S_{j}$ are viewed as virtual BSs, and $r_{i}$ and $r_{j}$ are the LOS TOAs between two virtual BSs and MSs. Assuming that the measurement is executed $N$ times during BS motion; thus, $N+2$ measurement equations can be designed to compute for the position of MS by utilizing the LS algorithm and by combining the reconstructed TOA.

$$
X=\left(A^{T} A\right)^{-1} A^{T} B
$$

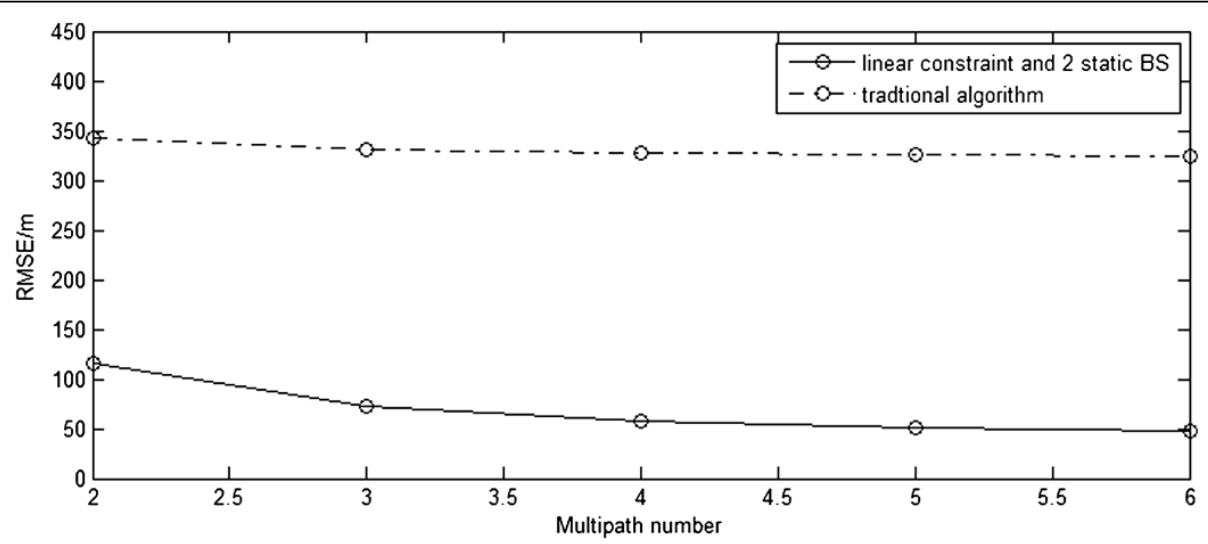

Figure 7 RMSE comparison of different numbers of multipath. 
Table 2 Simulation parameters

\begin{tabular}{ll}
\hline Coordinate of $\mathbf{M S}$ & $\mathbf{( 8 0 0 , 8 0 0 )}$ \\
\hline Velocity along the $x$-axis of BS & $0 \mathrm{~m} / \mathrm{s}$ \\
\hline Velocity along the $y$-axis of BS & $10 \mathrm{~m} / \mathrm{s}$ \\
\hline Simulation number & 2000 \\
\hline Multipath number & 2 \\
\hline
\end{tabular}

where

$$
\begin{aligned}
& X=\left[\begin{array}{l}
x \\
y
\end{array}\right], \\
& A=\left[\begin{array}{cc}
x_{S j}-x_{S i} & y_{S j}-y_{S i} \\
x_{S i}-x_{1} & y_{S i}-y_{1} \\
x_{1}-x_{2} & y_{1}-y_{2} \\
\vdots & \vdots \\
x_{N-1}-x_{N} & y_{N-1}-y_{N}
\end{array}\right] \text {, } \\
& B=\left[\begin{array}{c}
\frac{1}{2}\left[x_{S j}^{2}+y_{S j}^{2}-\left(x_{S i}^{2}+y_{S i}^{2}\right)+r_{i}^{2}-r_{j}^{2}\right] \\
\frac{1}{2}\left[x_{S i}^{2}+y_{S i}^{2}-\left(x_{1}^{2}+y_{1}^{2}\right)+\left(L_{1}^{L o s}\right)^{2}-r_{i}^{2}\right] \\
\frac{1}{2}\left[x_{1}^{2}+y_{1}^{2}-\left(x_{2}^{2}+y_{2}^{2}\right)+\left(L_{2}^{L o s}\right)^{2}-\left(L_{1}^{L o s}\right)^{2}\right] \\
\vdots \\
\frac{1}{2}\left[x_{N-1}^{2}+y_{N-1}^{2}-\left(x_{N}^{2}+y_{N}^{2}\right)+\left(L_{N}^{L o s}\right)^{2}-\left(L_{N-1}^{L o s}\right)^{2}\right]
\end{array}\right]
\end{aligned}
$$

\section{Simulation and analysis}

The improved algorithm in Section 3 is simulated and researched, and a macrocell district is assumed. BS1 is located in the original point of the Cartesian coordinate system, whereas BS2 is located in $(0,2000) \mathrm{m}$. BS is static and MS is moving. The remaining simulation parameters are shown in Table 1.

Figure 5 shows the mean-squared error (MSE) of the different TOA and AOA measurement errors in the disk of scatterers (DOS) model with a radius of $500 \mathrm{~m}$. The AOA measurement error has a greater influence on positioning accuracy than that of the TOA measurement error. Thus, the effect of the TOA measurement error is evident when the AOA measurement error is minimal. However, the positioning accuracy is mainly influenced by the AOA measurement error when the AOA measurement error is large.

Figure 6 shows the comparison of the root-meansquare error (RMSE) of the model with different radii. The standard TOA and AOA measurement errors are $1.5 \mathrm{~m}$ and $0.005 \mathrm{rad}$, respectively. This finding concludes that the performance of the improved algorithm is better than that of the traditional algorithm. The performance of the traditional algorithm degrades with increasing radius. Consequently, the improved positioning algorithm, which is based on linear constraints on scatterers in two BSs, performs better than the traditional algorithm. The positioning error of the proposed algorithm is also stable.

Figure 7 compares the positioning error of simulation parameters in the DOS model for different numbers of multipaths. Figure 7 concludes that the RMSE of positioning decreases with increasing number of multipath.

Simultaneously, we analyze the improved algorithm in Section 5, in which the BS is mobile and the MS is stationary. The simulation parameters are shown in Table 2. The distance of the two measurement points is $50 \mathrm{~m}$.

Figure 8 shows the RMSE of the positioning result under different TOA and AOA measurement errors.

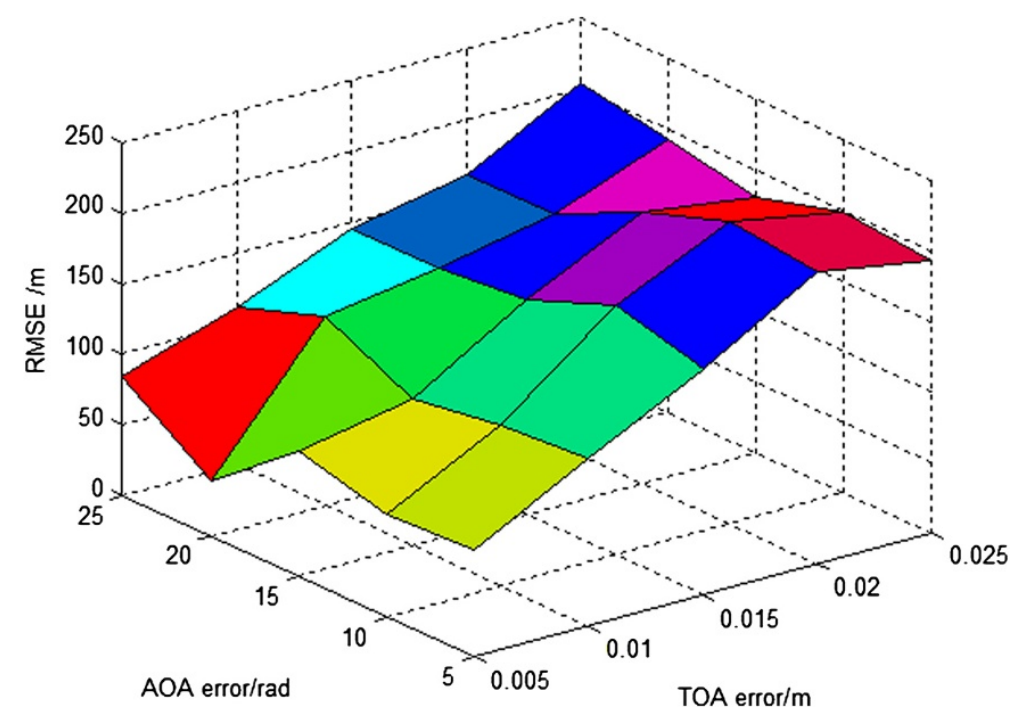

Figure 8 RMSE of different AOA and TOA measurement errors. 


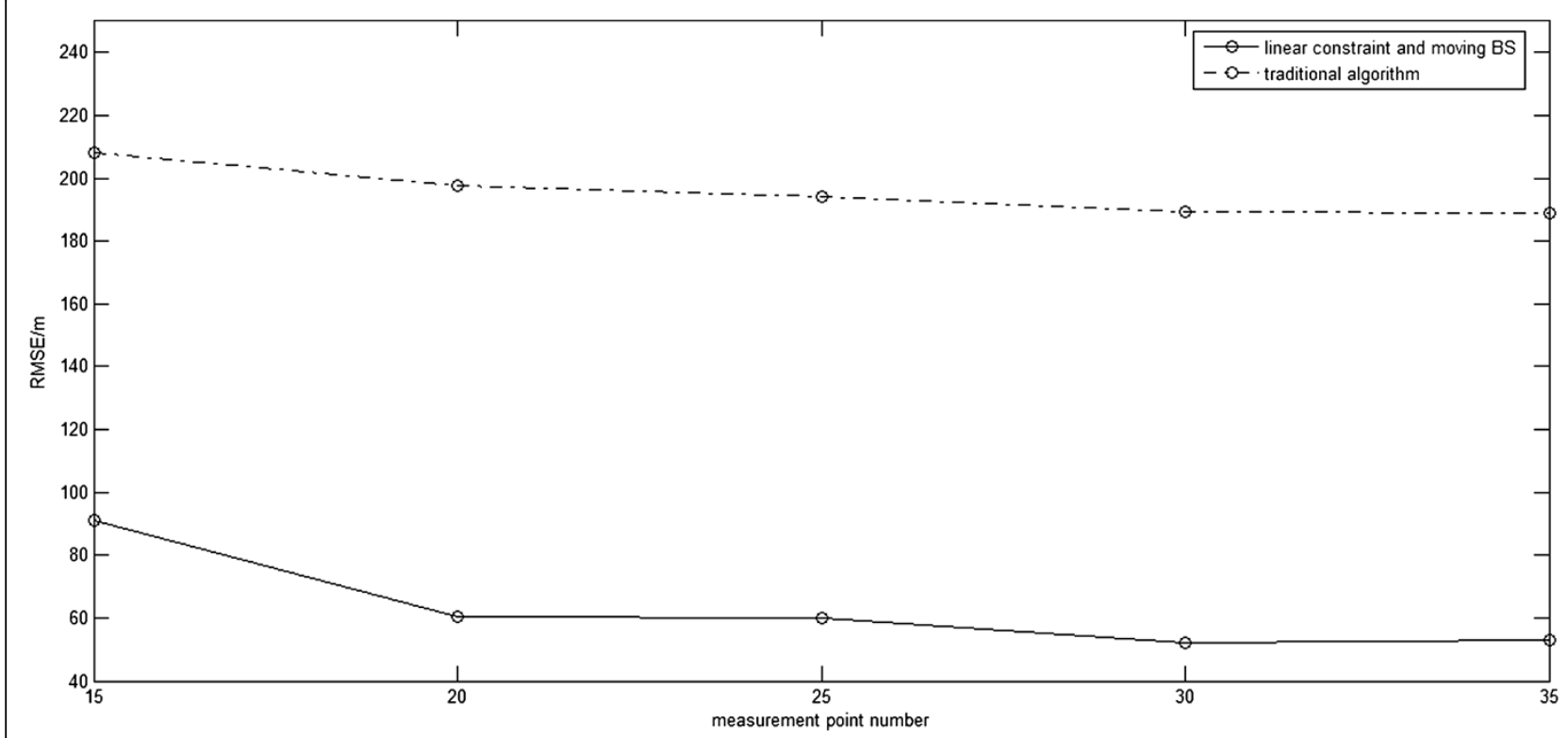

Figure 9 RMSE of different measurement point numbers.

Figure 8 denotes that the positioning accuracy is influenced mainly by the AOA measurement error, which agrees with the result of Figure 5 .

Figure 9 denotes that the improved algorithm for scatter linear restriction, which is based on mobile BS, performs better than the traditional algorithm. The performance of the above algorithms improves with increased measurement points because more geographic information is provided with more measurement points.

If the TOA measurement standard error is $1.5 \mathrm{~m}$, then the AOA measurement standard error is 0.005 rad. Table 3 shows the RMSE comparison of the traditional algorithm and the two improved algorithms proposed in this article.

This article proposes an improved traditional positioning algorithm by using scatter constraint information. The improved algorithm suits more common cases and performs better than the traditional algorithm. Further research is necessary to improve this study.

Table 3 RMSE comparison of different algorithms (m)

\begin{tabular}{llll}
\hline $\begin{array}{l}\text { Multipath } \\
\text { signal number }\end{array}$ & $\begin{array}{l}\text { Traditional } \\
\text { algorithm }\end{array}$ & $\begin{array}{l}\text { Improved } \\
\text { algorithm } \\
\text { based on } \\
\text { static BS }\end{array}$ & $\begin{array}{l}\text { Improved } \\
\text { algorithm } \\
\text { based on } \\
\text { moving BS }\end{array}$ \\
\hline 2 & Invalid & 116.15 & 77.75 \\
\hline 3 & Invalid & 71.70 & 46.14 \\
\hline 4 & 259.80 & 56.90 & 43.20 \\
\hline 5 & 116.62 & 51.60 & 40.23 \\
\hline
\end{tabular}

\section{Conclusion}

This article mainly analyzes the positioning algorithm based on linear constraint on scatterers and gives the drawback that the precondition of traditional algorithm is hard to be satisfied. So, an improved algorithm is proposed to reduce the impact of the drawback. This article first analyzes the key technology of the algorithm, such as multipath signal pairing and TOA reconstruction. Then in the algorithm proposed this article, the number of scatterer for positioning is reduced to two. The improvement can enhance the application range of algorithm, and would not deteriorate the positioning performance. Second, when MS is stationary, the proposed algorithm is adjusted to suit a special case which $\mathrm{BS}$ is moving. Both improved algorithms have the same advantage and the positioning performance. These algorithms mainly focus on the design of positioning algorithm, and the restriction of NLOS error. There are many other factors which influenced positioning accuracy, such as signal form and signal band. We will continue the research in the further work.

\section{Competing interests \\ The authors declare that they have no competing interests.}

\section{Acknowledgment}

This work was supported by the special fund of Chongqing key laboratory (CSTC).

Received: 5 December 2012 Accepted: 7 February 2013

Published: 21 March 2013 


\section{References}

1. JC Liberti, TS Rappaport, A geometrically based model for line-of-sight multipath radio channels, in Proceedings of the IEEE Vehicular Technology Conference, ed. by. Atlanta, USA, April 1996, Vol.2, pp. 844-848

2. MT Simsim, NM Khan, R Ramer, PB Rapajic, Time of arrival statistics in cellular environments, in IEEE 63rd Vehicular Technology Conference, VTC 2006-Spring, Melbourne, Australia, May, 2006, ed. by, 6th edn., pp. 2666-2670

3. P Petrus, JH Reed, TS Rappaport, Geometrical-based statistical macrocell channel model for mobile environments. IEEE Trans. Commun. 50(3), 495-502 (2002)

4. R Janaswamy, Angle and time of arrival statistics for the Gaussian scatter density model. IEEE Trans. Wirel. Commun. 1(3), 488-497 (2002)

5. YL Wu, KT Wong, A geometrical model for the toa distribution of uplink downlink multipaths assuming scatterers with a conical spatial density. IEEE Antennas Propagat. Mag. 50(6), 196-205 (2008)

6. J Zhou, L Qiu, C Li, H Kikuchi, Analyses and comparisons of geometricalbased channel model arisen from scatterers on a hollow-disc for outdoor and indoor wireless environments. IET Commun. 6(17), 2775-2786 (2012)

7. A Borhani, M Pätzold, Time-of-arrival, angle-of-arrival, and angle of-departure statistics of a novel simplistic disk channel model, in Proceedings of the 5th International Conference on Signal Processing and Communication Systems, ICSPCS, ed. by (, Honolulu, Hawaii, December, 2011), pp. 1-7

8. CS Wang, M Guo, KT Wong, Fourth-order spatial correlation-coefficient across the uplink receiver's spatial aperture-analytically derived in closed form. IEEE Trans. Commun. 60(3), 724-734 (2012)

9. N Thomas, DGM Cruickshark, DI Laurenson, Calculation of mobile location using scatter information. Electron. Lett. 37(19), 1193-1194 (2001)

10. Q Wan, WL Yang, YN Peng, Closed-form solution to mobile location using linear constraint on scatterer. Electron. Lett. 40, 883-884 (2004)

11. Y Xie, Y Wang, P Zhu, X You, Grid-search-based hybrid TOA/AOA location techniques for NLOS environments. IEEE Commun. Lett. 13(4), 254-256 (2009)

12. Y TianChi, J Liang, Single station location method in NLOS environment: the circle fitting algorithm. Sci. China: Inf. Sci. 54(2), 381-385 (2011)

13. J Zhou, C Li, L Qiu, H Kikuchi, Geometrical statistical channel model arisen from scatterers around the mobile with an inverted-parabolic spatial distribution. J. China Univ. Posts Telecommun. (English Edition) 19(5), 1-8 (2012)

14. KN Le, A new formula for the angle-of-arrival probability density function in mobile environment. Signal Process. 87(6), 1314-1325 (2007)

15. A Borhani, M Pätzold, A unified disk scattering model and its angle-ofdeparture and time-of-arrival statistics. IEEE Trans. Veh. Technol. 99, 1-12 (2013)

16. M Zhaounia, MA Landolsi, R Bouallegue, Hybrid TOA/AOA mobile localization with NLOS mitigation in ring scattering environments, in 3rd International Symposium on Wireless Pervasive Computing, ed. by (ISWPC 2008, Santorini, Greece, May, 2008), pp. 370-373

17. J Yan, L Wang, L Wu, Passive location estimation using scatterer information for non-line-of-sight environments. J. Southeast Univ. (English Edition) 26(4), 518-522 (2010)

18. H Miao, K Yu, M Juntti, Positioning for NLOS propagation: algorithm derivation and Cramer-Rao bounds. IEEE Trans. Veh. Technol. 56(5), 2568-2580 (2007)

19. X Wei, AOD/AOA/TOA-based 3D positioning in NLOS multipath environments, in IEEE 22nd International Symposium on Personal Indoor and Mobile Radio Communications (PIMRC), ed. by (, Toronto, Canada, September, 2011), pp. 1289-1293

20. P Meissner, T Gigl, K Witrisal, Scatterer and virtual source detection for indoor UWB channels, in IEEE International Conference on Ultra-Wideband (ICUWB), ed. by (, Bologna, Italy, September, 2011), pp. 14-16

21. S Venkatraman, JR James Caffery, A statistical approach to non-line-of-sight BS identification, in IEEE Wireless Personal Multimedia Communications. (WPMC) Conference, ed. by, 1st edn. (, Honolulu, Hawaii, October, 2002), pp. 296-300

22. TC Yang, CQ Yu, TP Wang, L Jin, A single observer location method under the scatter signals position disturbance situation: the constraint total least square method. Sci. China: Inf. Sci. 54(1), 146-152 (2011)

\section{doi:10.1186/1687-1499-2013-84}

Cite this article as: Zhou et al:: Improved positioning algorithm based on linear constraints on scatterers. EURASIP Journal on Wireless Communications and Networking 2013 2013:84.

\section{Submit your manuscript to a SpringerOpen ${ }^{\circ}$ journal and benefit from:}

- Convenient online submission

- Rigorous peer review

- Immediate publication on acceptance

- Open access: articles freely available online

- High visibility within the field

- Retaining the copyright to your article

Submit your next manuscript at $>$ springeropen.com 